\title{
Trait anxiety, infrequent emotional conflict, and the emotional face Stroop task.
}

\section{Andrew R. du Rocher and Alan D. Pickering.}

\begin{abstract}
Research shows that anxiety may relate to any or all of the following: goal conflict resolution; distraction, and the automatic detection of threat-related stimuli. To investigate these relationships we used a modified Stroop task where fearful and happy emotional target faces are overlaid with either emotionally neutral, emotionally congruent or emotionally incongruent distracting words. A trait anxiety related speed-accuracy trade-off seemed to primarily reduce accuracy during incongruent trials with target fearful faces overlaid with the emotionally conflicting word happy. We offer an explanation of this effect based upon theories of how positive stimuli and threat-related stimuli differentially affect information processing. Future studies should seek to verify how the anxiety related speed-accuracy trade-off mechanism is activated, and elucidate how and when positive stimuli affect anxiety more than threat-related stimuli do.
\end{abstract}

Keywords: Anxiety, conflict, distraction, emotion, attention, Stroop.

\subsection{Introduction}

Theories of trait anxiety have proposed distinct, but conceptually-related, mediating mechanisms. Firstly, anxiety may be mediated by a biological behavioural inhibition system 
which is responsible for the resolution of goal conflict (Gray \& McNaughton, 2000).

Secondly, attentional control theory (ACT) suggest that anxiety relates to increased cognitive interference, which is experienced as distraction (Eysenck, Derakshan, Santos \& Calvo, 2007). Thirdly, anxiety has been related to the enhanced processing of threat-related stimuli (Bar-Haim, Lamy, Pergamin, Bakermans-Kranenburg, \& van Ijzendoorn, 2007; Beck \& Clark, 1997; Mathews \& Mackintosh, 1998; Mogg \& Bradley, 1998). Therefore, the day-today experiences of those high in trait anxiety may be affected by the interaction between the level of threat monitoring, magnitude of cognitive interference (i.e., distraction), and an altered way of resolving conflicting approach and avoidance situations.

Experimental neurocognitive research has shown that trait anxiety is related to the impaired reactivity of cognitive mechanisms that are mediated by the prefrontal cortex, when goal conflict resolution and the inhibition of distractor processing is required. These effects have been found when neutral distractors are used (Bishop, 2009), and when threat-related distractors are used (Bishop, Jenkins, \& Lawrence, 2007). Bishop (2009) proposed that these findings may explain why clinical anxiety patients suffer daily problems with concentration. A widely used emotional distraction paradigm referred to as the emotional Stroop task requires participants to identify the font colour of threat-related words and neutral words. If the font colour of the threat-related words is identified slower than the font colour of the neutral words, a threat-related attentional bias is inferred. This bias is magnified in anxiety (for meta-analysis see Phaf \& Kan, 2007).

The emotional Stroop task has recently received a substantial paradigm modification. In this paradigm distracting emotional words (e.g., fear and happy) are overlaid upon pictures of 
either fearful faces or happy faces. Participants are required to respond to the faces. The distracting emotional words are either congruent or incongruent with the emotional expressions of the target faces. Goal conflict is high during incongruent trials (e.g., a happy face with a fearful distractor word) as the distractor words may trigger their own response. Goal conflict is low during congruent trials as the distractor words trigger the same response as that of the target facial expression. A congruency effect is calculated as the reaction time (RT) or accuracy difference between the faster and more accurate responses to congruent trials and slower and less accurate responses to incongruent trials. Thus, this paradigm can be used to assess how trait anxiety affects attentional control in situations of emotional conflict. Etkin \& Schatzberg (2011) and Etkin, Prater, Hoeft, Menon \& Schatzberg (2010) used this paradigm including equal proportions of congruent and incongruent trials. The congruency effect was not related to levels of trait anxiety or clinical anxiety. These data are at odds with the proposed theoretical link between anxiety and goal-conflict resolution. However, Etkin and colleagues did not analyse whether anxiety selectively affected performance on either incongruent happy trials, or incongruent fearful trials.

Krug \& Carter (2010) also used this paradigm but they used fearful and neutral faces overlaid with the words fear or neutral, and included just $30 \%$ incongruent trials. Therefore, in this study goal conflict and distraction occurred less frequently. Trait anxiety still did not affect the congruency effect. However, high anxious participants were slower to respond to neutral faces overlaid with the distractor word fear, than fearful faces overlaid with the distractor word neutral. Moreover, low anxious participants showed the opposite pattern. Therefore these results favour an account of anxiety which tentatively suggests that high anxiety relates to increased automatic threat detection. Krug \& Carter (2012) used the same task again. In a high conflict expectancy condition $65 \%$ of trials were incongruent, whereas in a low conflict 
expectancy condition $35 \%$ of trials were incongruent. However, this time neither the congruency effects, nor performance during incongruent trials were correlated with trait anxiety.

In the word-face Stroop studies discussed above, goal conflict was induced between facial and verbal emotional stimuli, with a view to quantifying how trait anxiety effects the cognitive control of emotion. These four studies make it clear that support for the three theories of anxiety discussed above was very inconsistent. It is possible that the task designs used did not cleanly measure the theoretical processes being discussed here. For example, in the real world emotional conflict/distraction may occur less frequently. Moreover, congruency effects in Stroop tasks are the sum of both facilitation effects and interference effects. Facilitation effects are measured as the improvement in performance for congruent trials relative to neutral trials, whereas interference effects are measured as the reduction in performance for incongruent trials relative to neutral trials (Goldfarb \& Henik 2007). Koster, Crombez, Verschuere, \& De Houwer (2004) have shown that both facilitation and interference effects also contribute to the congruency effect found in the dot probe paradigm. This suggests that the measurement of attentional facilitation and attentional interference may generalise across paradigms. The differential contributions of these effects were not assessed in the word-face Stroop studies discussed above. Thus, any specific trait anxiety effects on attentional facilitation or attentional interference were not determined.

Decreasing the proportion of incongruent trials relative to congruent trials increased the magnitude of the congruency effect in the emotional word-face Stroop study by Krug \& Carter (2012). Current theorising suggests that participants would have implicitly learned the correlations (contingencies) between the words and responses. Thus participants would have 
learned to predict the response required based upon the distracting word (Schmidt \& Besner, 2008). Schmidt \& Besner suggested that in Stroop tasks participants make speeded responses to high contingency congruent trials based upon response predictions. They proposed that participants selectively lower their response threshold for an expected response. Schmidt \& Besner suggested that increased RT facilitation, but not increased RT interference, is what increases the magnitude of the RT congruency effect, when predominantly congruent trials are included. They also suggest that increased facilitation in accuracy occurs due to the same mechanism. Moreover, interference effects in accuracy for low contingency incongruent trials also occur due to the response threshold being lowered for the word when it was predictive. If this theoretical framework better describes proportion congruent effects in Stroop tasks, then this suggests that there is an extra neurocognitive mechanism that may be implicated, in addition to any conflict resolution, distraction, and threat detection mechanisms. Thus, any anxiety effects found might be based upon contingency learning and/or response threshold lowering.

\subsection{Purpose of study}

None of the word-face emotional Stroop studies discussed above used a design that combined conflicting fearful and happy stimuli with infrequent incongruent trials. Thus, we used a happy versus fear word-face Stroop task including very infrequent incongruent trials (17\%), in an attempt to maximise the effect of emotional conflict during these trials. We included neutral word trials so we could explore whether trait anxiety differentially modulated how attentional facilitation or attentional interference contributed to the congruency effect. 
Specifically, we used the above theories to predict that trait anxiety would impair cognitive performance during the very infrequent incongruent trials, resulting in either an anxiety related slowing in RTs and/or reduced accuracy for these trials (or an increased congruency effect). This prediction is based upon the suggestion that high anxiety is related to increased cognitive interference/distraction (Eysenck et al., 2007), and also the impaired prefrontal control of attention (Bishop, 2009; Bishop et al., 2007). We predicted that any anxiety effect upon incongruent trials would be magnified for incongruent trials consisting of happy faces overlaid with the word "fear" more so than for fearful faces overlaid with the word "happy". We predicted this based on the theoretical view that distraction should be maximized in high anxiety, especially when the distracting stimuli are threat related (Bar-Haim et al., 2007;

Beck \& Clark, 1997; Eysenck et al., 2007; Mathews \& Mackintosh, 1998; Mogg \& Bradley, 1998). If found, this result would be consistent with the results of Krug \& Carter (2010).

\subsection{Method}

\subsection{Participants}

Participants ( $\mathrm{N}=77 ; 62$ female) were recruited from Goldsmiths University of London, and had a mean age of 23.4 years $(\mathrm{SD}=7)$. Of these, 68 were right-handed and 35 were psychology $1^{\text {st }}$ year undergraduates, who took part in return for course credit. The rest were paid $£ 5$, and were students or staff from other departments. All gave informed written consent in accordance with standard ethical guidelines. The approximate sample size was chosen to allow $80 \%$ power for two-tailed tests at $\mathrm{p}=0.05$, for correlations of 0.3 . Such correlations are typical for personality-behaviour associations. 


\subsection{Psychometric measures}

Trait anxiety was assessed using the State-Trait Anxiety Inventory (STAI, Spielberger et al., 1983).

\subsection{Stimuli}

The emotional faces used to create the Stroop stimuli were obtained from a standardised face stimuli set developed for research (NimStim; Tottenham et al., 2009). Two face stimulus sets were created using one male face, and one female face. This was an attempt to control for gender differences. Congruent stimuli were created by placing the distractor word "fear" or "happy" across the nose of the target fearful or happy faces, respectively. The mouths and eyes were not obscured. Incongruent stimuli were created by placing the distractor words "fear" or "happy" over the top of the happy or fearful faces, respectively. Neutral stimuli were created by placing either the words "bowl" or "cellar" over the emotional faces. The overall picture dimensions were $65 \mathrm{~mm}$ high and $53 \mathrm{~mm}$ wide, and were presented on a 15.5 inch laptop screen.

\subsection{Procedure}

Participants were told that they would be presented with two facial emotion recognition tasks (i.e., one male block of trials and one female block of trials) with a short rest in-between. Participants were asked to sit as close to the screen as was comfortable for their eyes (typical viewing distance was approximately $70 \mathrm{~cm}$ ). The task instructions were presented on the screen. To start each block the first screen instructed participants that they would have to judge the emotional expression showing on photos of happy or fearful faces. Participants 
were then shown examples of the various stimulus combinations they might see and reminded to concentrate on the face and ignore the words. They were told to rest their index fingers over the responses keys ( $\mathrm{z}$ and /) and to respond as fast as possible while maintaining high accuracy levels. They were verbally told that a high pitched tone following a response indicates a correct response, whereas a low pitched tone following a response indicates an incorrect response.

The experimental stimuli were displayed until a response key was pressed. Unbeknown to the participants, at the beginning of each block, there were two of each neutral and incongruent trials, and 8 of each congruent trial included as practice trials; these were discarded and not analysed. The main experimental stimuli that followed consisted of 40 neutral trials, 40 incongruent trials and 160 congruent trials (in each of the two blocks). The proportions of target happy trials and target fearful trials were kept equal. The trial type sequence was created using a random number generator, and was the same for all participants. We kept the sequence the same for all participants as this is an individual differences study, and we wanted as few uncontrolled variables as possible to vary across participants. We also used the same trial type sequence for each of the blocks. There were 240 non-practice trials in total in each block so we felt that there was no chance that using the same sequence in each block would cause any learning of the sequence of trial types. We counterbalanced the order of the 2 blocks across participants. Each block lasted for approximately ten minutes. The left/right finger response key mappings were also counterbalanced. The data for each trial type in the two blocks was averaged prior to analysis. 


\subsection{Results}

\subsection{Psychometric measurement}

Participants trait anxiety scores ranged from 20-70 (mean $=41.7, \mathrm{SD}=10.2)$.

\subsection{Reaction times}

RTs for correct trials (excluding trials where RT $<250$ msecs and RT $>1000$ msecs) were first subjected to a repeated measures $3 \times 2$ ANCOVA with factors of trial type (congruent versus neutral versus incongruent) and emotion (fearful face versus happy face) with standardised trait anxiety as the covariate. The descriptive statistics for the 6 stimulus types are shown in Table 1.

\section{TABLE 1}

The results of the within-subjects part of our ANCOVA directly address the theories being tested here as they reflect the effects of trait anxiety on the congruency effect and its components. The test of within-subjects effects showed that the main effect of trial type was significant $\left(F[2,144]=15.1, p<0.001, \eta^{2}=0.17\right)$. The critical finding here was that anxiety did not did not modulate the main effect of trial type $\left(F[2,144]=1.6, p=0.21, \eta^{2}=0.022\right)$. The effect of emotion was also non-significant $\left(F[1,72]=1.23, p=0.27, \eta^{2}=0.017\right)$, and critically did not interact with anxiety $\left(F[1,72]=0.13, p=0.72, \eta^{2}=0.002\right)$. The effect of trial type did not further interact with emotion $\left(F[2,144]=0.46, p=0.63, \eta^{2}=0.006\right)$, and there was no significant three way interaction between trial type, emotion and anxiety $(F[2,144]=0.29, p=0.75$, $\left.\eta^{2}=0.004\right)$. 
We conducted three planned comparisons to clarify the main effect of trial type using an adjusted significance level of 0.05/3. A one-way ANCOVA with standardised trait anxiety as the covariate showed that congruent trials were responded to significantly faster than incongruent trials $\left(F[1,72]=22.1, p<0.001, \eta^{2}=0.235\right)$. This effect was unrelated to anxiety $\left(F[1,72]=0.09, p=0.76, \eta^{2}=0.001\right)$. This confirms that anxiety was uncorrelated with the congruency effect (incongruent RT minus congruent RT). Our next one-way ANCOVA with standardised trait anxiety as the covariate showed that congruent trials were responded to significantly faster than neutral trials $\left(F[1,72]=25.9, p<0.001, \eta^{2}=0.265\right)$. This effect was unrelated to anxiety $\left(F[1,72]=2.3, p=0.13, \eta^{2}=0.031\right)$. This confirms that anxiety was uncorrelated with the facilitation effect (neutral RT minus congruent RT). Our final one-way ANCOVA with standardised trait anxiety as the covariate showed that incongruent trials were not responded to significantly slower than neutral trials $\left(F[1,72]=1.4, p<0.711, \eta^{2}=0.002\right)$. This comparison was unrelated to anxiety $\left(F[1,72]=2.6, p=0.112, \eta^{2}=0.035\right)$. This confirms that anxiety was uncorrelated with the overall non-significant interference effect (incongruent RT minus neutral RT).

The results of the between-subjects part of the ANCOVA revealed interesting significant results. The test of between-subjects effects was significant $\left(F[1,72]=6.45 p=0.01, \eta^{2}=0.082\right)$, indicating that anxiety was significantly correlated with RTs across the whole experiment (the correlation value was $r=-0.3$ ). This is shown in figure 1 . We confirmed that this negative correlation was present for both target fearful faces $(r=-0.29, p=0.012)$, and target happy faces $(r=-0.31, p=0.007)$ considered separately. These correlations are significant at an adjusted significance level of 0.025 . 


\section{FIGURE 1}

\subsection{Proportion correct}

We analysed the proportion correct for each of the trial types (excluding trials where RT < 250 msecs and RT > 1000 msecs) using a $3 \times 2$ ANCOVA with factors of trial type (congruent versus neutral versus incongruent) and emotion (fearful face versus happy face) with standardised trait anxiety as the covariate. The descriptive statistics for the 6 stimulus types are shown in Table 2.

TABLE 2

The test of within-subjects effects showed that the main effect of trial type was significant $\left(F[2,144]=24.6, p<0.001, \eta^{2}=0.255\right)$. Anxiety significantly modulated the trial type effect $\left(F[2,144]=3.0, p=0.054, \eta^{2}=0.040\right)$. There was no significant main effect of emotion $\left(F[1,72]=1.06, p=0.307, \eta^{2}=0.014\right)$, and no anxiety interaction $(F[1,72]=1.70, p=0.194$, $\left.\eta^{2}=0.023\right)$. The non-significant emotion versus anxiety interaction shows that anxiety does not correlate with the accuracy difference score between happy and fearful target trials. The effect of trial type significantly interacted with emotion $\left(F[2,144]=4.74, p=0.010, \eta^{2}=0.062\right)$, but there was no significant three-way interaction between trial type, emotion and anxiety $\left(F[2,144]=0.30, p=0.74, \eta^{2}=0.004\right)$. 
We followed up the anxiety by trial type effect using three one-way ANCOVAs with standardised trait anxiety as the covariate. Here we adopt an adjusted significance level of 0.05/3. A traditional congruency effect was evident as congruent trials were responded to more accurately than incongruent trials $\left(F[1,72]=27.2 p<0.001, \eta^{2}=0.274\right)$. Anxiety was related to this difference in accuracy at a trend level $\left(F[1,72]=3.67 p=0.059, \eta^{2}=0.048\right)$. The sign and value of the correlation was $r=0.22$. Thus, as anxiety increased so did the congruency effect. Congruent trials were not responded to significantly more accurately than neutral trials $\left(F[1,72]=0.10 p=0.749, \eta^{2}=0.001\right)$, and this comparison did not interact with anxiety $\left(F[1,72]=0.37 p=0.543, \eta^{2}=0.005\right)$. Therefore, there were no facilitation effects in the accuracy data. However, responses to neutral trials were more accurate than responses to incongruent trials $\left(F[1,72]=27.56 p<0.001, \eta^{2}=0.277\right)$. This interference effect was not significantly related to anxiety, although it could be considered a weak trend $(F[1,72]=2.85$ $\left.p=0.096, \eta^{2}=0.038\right)$.

As the anxiety and trial type analyses focus upon the difference between trial types we wished to determine if anxiety correlated with accuracy during each of the 3 trial types separately. Trait anxiety was related to reduced accuracy for incongruent trials $(r=-0.26$, $p=0.024$ ). This can be interpreted as a trend against an adjusted significance level of 0.05/3. Anxiety was not significantly correlated with accuracy to congruent trials $(r=-0.12, p=0.307)$ or neutral trials $(r=-0.17, p=0.144)$.

As with the RT analysis, the test of between-subjects effects revealed an interesting and significant result, not directly predicted by the theories under investigation $(F[1,72]=4.79$ 
$p=0.032, \eta^{2}=0.062$ ). Thus, anxiety was significantly correlated with proportion correct across the whole experiment (the correlation value was $r=-0.25$; as shown in figure 2). Thus the overall speeding in RTs by high anxious participants also seemed to result in an overall reduction in accuracy.

\section{FIGURE 2}

We then verified whether both averaged fear targets and averaged happy targets, or if only one of the averaged target emotion types were implicated in this anxiety related reduction in accuracy. Here we adopt an adjusted significance level of 0.025. Correlations showed that anxiety negatively and significantly correlated with proportion correct for fear trials $(r=$ $0.29, p=0.013)$, but not happy trials $(r=-0.16, p=0.185)$. We wished to determine which of the fear trials were driving the anxiety and fear trial correlation. We conducted exploratory correlations between anxiety and the three fear trial types (i.e., incongruent, neutral and congruent). As figure 3 shows, anxiety was more robustly correlated with incongruent fear trials $(r=-0.28, p=0.017)$, than neutral fear trials $(r=-0.17, p=0.149)$, or congruent fear trials $(r=-0.17, p=0.138)$.

\section{FIGURE 3}

\subsection{Speed-accuracy trade-off}

A reliable speed accuracy trade-off was present, as overall RTs correlated positively with overall proportion correct $(r=0.39, p=0.001)$. As trait anxiety was significantly correlated 
with both an overall decrease in RTs, and an overall decrease in accuracy, we considered that this was likely to reflect an anxiety related speed-accuracy trade-off. To verify this we correlated trait anxiety with the overall proportion correct across the experiment whilst controlling for overall RTs. When partialling out the effects of RTs, trait anxiety was no longer significantly correlated with proportion correct $(r=-0.15, p=0.20)$. Moreover, the correlation between trait anxiety and RTs was no longer reliable when controlling for accuracy $(r=-0.22, p=0.06)$. We suggest that these partial correlations indicate an anxiety related speed-accuracy trade-off.

\subsection{Discussion}

We used a word-face emotional Stroop task which produced a robust RT congruency effect and RT facilitation effect, but no RT interference effect. Our accuracy analysis showed a robust congruency effect, robust interference effect, but no facilitation effect. Our RT data lends some support to the contingency learning theory proposed by Schmidt and Besner (2008), which predicts that the RT congruency effect in contingency biased designs (i.e., those including predominantly congruent trials) will be driven mainly by facilitation not interference. Our accuracy data only partially supports the contingency learning account, as although the expected interference effects were found, the expected facilitation effects were not found. In other words, we found reduced accuracy for incongruent trials relative to neutral trials, but no increase in accuracy for congruent trials relative to neutral trials. However, Schmidt and Besner used equiprobable contingency trials (50\% Incongruent and $50 \%$ congruent) to compare to high and low contingency trials (and thus separate facilitation and interference effects differently). In contrast, we used neutral word trials (in the same proportion as incongruent trials) that were not predictive or emotionally conflicting. 
In the present study, the effect of anxiety predicted overall for incongruent trials, was not found for RTs, but was marginally present for accuracy. Thus, our initial analyses suggested that trait anxiety was not reliably related to increased cognitive interference/distraction, which is somewhat inconsistent with ACT (Eysenck et al., 2007). Moreover, we found no evidence of an anxiety related attentional bias towards threat-related stimuli, which is inconsistent with several theoretical perspectives on anxiety (Bar-Haim et al., 2007; Beck \& Clark, 1997; Eysenck et al., 2007; Mathews \& Mackintosh, 1998; Mogg \& Bradley, 1998). In contrast to the above, the present study found that trait anxiety was related to faster RTs across the whole paradigm, which was related to an overall reduction in accuracy (i.e., a speed accuracy trade-off). However, further analyses suggested that there was actually a more specific effect of anxiety upon cognitive interference/distraction and emotion processing. Anxiety was robustly related to reduced accuracy for fearful trials, which seemed primarily due to the incongruent fearful trials (a fearful target face overlaid with the word happy). We now offer a novel explanation of the effects of anxiety upon the speed accuracy trade-off, and the resulting decrement in accuracy for incongruent fearful trials.

The finding of an anxiety-related speed-accuracy trade-off is in alignment with the results reported by Basten, Stelzel, \& Fiebach (2011). They found that high anxiety was related to a trend towards an overall speeding in RTs, and increased errors in a neutral Stroop task. It is possible that both the present study, and the study carried out by Basten et al., may have tapped into an anxiety related speed-accuracy trade-off mechanism. The question remains open as to what triggered the speed-accuracy trade-off, and what made it greater in high anxious participants, in the present study. Van Veen, Krug, and Carter (2008) proposed that 
when a stimulus is perceived, evidence is processed that relates to each possible response. They suggest that neural activity relevant to making a possible response will start from baseline and gradually increase until a response threshold is reached. Schmidt and Besner (2008) suggest that in Stroop tasks participants make speeded responses to high contingency trials based upon response predictions. They suggest that participants lower a response threshold for expected responses, but not for any alternative possible responses. They also suggest that increased interference during low contingency trials will produce more errors, as the response threshold was reduced for the word because it was previously predictive.

In the present study anxiety did not modulate any RT facilitation effects for congruent trials. Thus we suggest that the contingency learning effect was of the same magnitude at all levels of anxiety. However, we suggest that contingency learning may still have been the trigger for the speed-accuracy trade-off, and its increase in high anxious participants. The anxiety related speed-accuracy trade-off was much stronger in the present study than the trend in the study by Basten et al. (2011). In their study their results may have reflected an underlying response tendency that was not yet fully triggered. Indeed, in their study no contingency learning would have been present as congruent and incongruent trials were equal in proportions.

In the present study the main detrimental effects of the anxiety related speed-accuracy tradeoff seemed to be a reduction in accuracy during incongruent trials requiring responses to target fearful faces (overlaid with the distractor word happy). The question is, why in the present study was it harder for high anxious participants to suppress responses to happy words than fear words during incongruent trials? We suggest that it is the combination of two theoretical factors that can explain these results. Firstly, a general attentional bias to positive 
stimuli may occur at an initial attentional orienting stage of information processing (Pool, Brosch, Delplanque, \& Sander 2016). Secondly, anxiety may specifically relate to a difficulty in disengaging attention from threat-related stimuli that occurs at a later stage of information processing (e.g., Phaf \& Kan, 2007; Yiend \& Mathews, 2001).

According to these two perspectives, in the present study all participants' attention to the target happy faces and happy distractor words would have been affected at an early orienting stage of information processing. However, a delayed attentional disengagement from target fearful faces and fearful distractor words, by high anxious participants, would occur at a slightly later stage of information processing. In the present study the initial orienting to happy words, their learned contingencies, and the anxiety related speed-accuracy trade-off could have made it particularly hard for those high in anxiety, to inhibit responding to the happy words. This may explain the anxiety related decrement in accuracy for incongruent fearful trials. In short, the target fearful faces may not have had the chance to affect attention (and thus the responses) of those high in anxiety, if the responses to happy words were prepared exceptionally quickly.

In the present study anxiety did not relate to reduced accuracy during incongruent trials consisting of target happy faces overlaid with fearful words. We suggest that the fearful words should have affected the attention of high anxious participants at the later disengagement stage. However, in all participants the happy target faces would have affected attention at the initial orienting stage. Thus, even though responses to the fearful words would have been contingency learned, it may have been slightly easier for high anxious participants to respond to the target happy face by inhibiting responding to the fearful word (during 
incongruent happy trials), than it would be for them to respond to the target fearful face by inhibiting responding to the happy word (during incongruent fear trials). In short, we suggest that the different effects of the two types of incongruent trials, upon those high in anxiety, was due to differences in when and how their attention was affected by the differentially valenced emotional stimuli.

\subsection{Limitations}

In the present study female participants outnumbered male participants. A lot of research with UK psychology student samples has this bias, as females outnumber males on UK psychology degrees three or four to one. We are not overly concerned by this issue in the present study for two reasons. Firstly, MacLeod (1991) reviewed Stroop studies spanning half a century and reported that research has not found any reliable gender difference in Stroop task performance. Secondly, Hoffmann, Kessler, Eppel, Rukavina, \& Traue (2010) have shown that there is no gender difference in recognition accuracy for salient emotional facial expressions.

\subsection{Conclusion}

To conclude, we used a contingency biased emotional word-face Stroop task that resulted in an anxiety-related speed-accuracy trade-off. This resulted in an anxiety-related reduction in accuracy for incongruent trials. However, anxiety seemed to be mainly related to increased interference by positive emotional words as opposed to increased interference by threatrelated words. Future studies should seek to ascertain if the anxiety related speed-accuracy trade-off is replicable, and further explain how this mechanism is triggered. In addition, 
future studies should seek to further elucidate how and when positive emotional stimuli might affect high trait anxious people more than threat-related stimuli do.

\section{References}

Bar-Haim, Y., Lamy, D., Pergamin, L., Bakermans-Kranenburg, M, J., \& van Ijzendoorn, M, H. (2007). Threat-related attentional bias in anxious and nonanxious individuals: A meta-analytic study. Psychological Bulletin, 113, 1, 1-24. DOI: 10.1037/00332909.133.1.1

Basten, U., Stelzel, C., \& Fiebach, C. J. (2011). Trait anxiety modulates the neural efficiency of inhibitory control. Journal of Cognitive Neuroscience, 23:10, 3132-3145. DOI:10.1162/jocn_a_00003

Beck, A. T., \& Clark, D. A. (1997). An information processing model of anxiety: Automatic and strategic processes. Behaviour Research and Therapy, 35, 49-58. DOI.org/10.1016/S0005-7967(96)00069-1

Bishop, S. J. (2009). Trait anxiety and impoverished prefrontal control of attention. Nature Neuroscience, 12, 1, 92-98. DOI: 10.1038/nn.2242

Bishop, S. J., Jenkins, R., \& Lawrence, A. D. (2007). Neural processing of fearful faces: Effects of anxiety are gated by perceptual capacity limitations. Cerebral Cortex, 17, 1595-1603. DOI:10.1093/cercor/bhl070 
Etkin, A., \& Schatzberg, A, F. (2011). Common abnormalities and disorder-specific compensation during implicit regulation of emotional processing in generalised anxiety and major depressive disorders. American Journal of Psychiatry, 168, 968978. DOI:10.1176/appi.ajp.2011.10091290

Etkin, A., Prater, K, E., Hoeft, F, H., Menon, V., \& Schatzberg, A, F. (2010). Failure of anterior cingulate activation and connectivity with the amygdala during implicit regulation of emotional processing in generalised anxiety disorder. American Journal of Psychiatry, 167, 545-554. DOI:10.1176/appi.ajp.2009.09070931

Eysenck, M, W., Derakshan, N., Santos, R., Calvo, M, G. (2007). Anxiety and cognitive performance: Attentional control theory. Emotion, 7, 2, 336-353. DOI:10.1037/15283542.7.2.336

Gray J, A. \& McNaughton, N. (2000).The neuropsychology of anxiety: an inquiry into the function of the septo-hippocampal system. Oxford University Press.

Goldfarb, L., \& Henik, A. (2007). Evidence for task conflict in the Stroop effect. Journal of Experimental Psychology: Human Perception and Performance, 33, 5, 1170-1176. DOI: $10.1037 / 0096-1523.33 .5 .1170$

Hoffmann, H., Kessler, H., Eppel, T., Rukavina, S., \& Traue, H. C. (2010). Expression intensity, gender and facial emotion recognition: Women recognise only subtle facial emotions better than men. Acta Psychologica, 135, 278-283.

DOI:10.1016/j.actpsy.2010.07.012

Koster, E. H. W., Crombez, G., Verschuere, B., \& De Houwer, J. (2004). Selective attention to threat in the dot probe paradigm: differentiating vigilance and difficulty to disengage. Behaviour Research and Therapy, 42, 1183-1192.

DOI:10.1016/j.brat.2003.08.001 
Krug, M, K. \& Carter, C, S. (2010). Adding fear to conflict: A general purpose cognitive control network is modulated by trait anxiety. Cognitive, Affective, \& Behavioural Neuroscience, 10, 3, 357-371. DOI:10.3758/CABN.10.3.357

Krug, M, K. \& Carter, C, S. (2012). Proactive and reactive control during emotional interference and its relationship to trait anxiety. Brain Research, 1481, 13-36. DOI:10.1016/j.brainres.2012.08.045

MacLeod, C. M. (1991). Half a century of Research on the Stroop effect: An integrative review. Psychological Bulletin, 109, 2, 163-203.

Mathews, A., Mackintosh, B. (1998). A cognitive model of selective processing in anxiety. Cognitive Therapy and Research, 22, 539-560. DOI:10.1023/A:1018738019346

Mogg, K., \& Bradley, B. P. (1998). A cognitive-motivational analysis of anxiety. Behaviour Research and Therapy, 36, 809-848. DOI.org/10.1016/S0005-7967(98)00063-1

Phaf, R. H., \& Kan, K-J. (2007). The automaticity of emotional Stroop: A meta-analysis. Journal of Behaviour Therapy and Experimental Psychiatry, 38, 184-199. DOI:10.1016/j.jbtep.2006.10.008

Pool, E., Brosch, T., Delplanque, S., \& Sander, D. (2016). Attentional bias for positive emotional stimuli: A meta-analytic investigation. Psychological Bulletin, 142, 1, 79106. DOI:10.1037/bul0000026

Schmidt, J. R. \& Besner. D. (2008). The Stroop effect: Why proportion congruent has nothing to do with congruency and everything to do with contingency. Journal of Experimental Psychology: Learning, Memory, and Cognition, 34, 3, 514-523. DOI: $10.1037 / 0278-7393.34 .3 .514$ 
Spielberger, C. D., Gorsuch, R. L., Lushene, R., Vagg, P. R., \& Jacobs,G. A. (1983). Manual for the State-Trait Anxiety Inventory. Palo Alto, CA: Consulting Psychologists Press.

Tottenham, N., Tanaka, J. W., Leon, A. C., McCarry, T., Nurse, M., Hare, T. A., et al. (2009). The NimStim set of facial expressions: judgments from untrained research participants. Psychiatry Research. 168, 242-249.

DOI:10.1016/j.psychres.2008.05.006.

van Veen, V., Krug, M, K., \& Carter, C, S. (2008). The neural and computational basis of controlled speed-accuracy tradeoff during task performance. Journal of Cognitive Neuroscience, 20:11, 1952-1965. DOI:10.1162/jocn.2008.20146

Yiend, J. \& Mathews, A. (2001). Anxiety and attention to threatening pictures. Quarterly Journal of Experimental Psychology: Human Experimental Psychology, 3, 665-681. DOI:10.1080/02724980042000462 
Tables \& Figures:

Table 1: Mean reaction times, $95 \%$ confidence intervals (95\%CI) and standard errors of the mean (SE) for each stimulus type. All are given in milliseconds.

Happy target faces $\quad$ Fearful target faces

\begin{tabular}{|c|c|c|c|c|c|c|c|}
\hline$\underline{\text { Trial type }}$ & Mean RT & $\underline{95 \% \mathrm{CI}}$ & $\underline{\underline{\mathbf{S E}}}$ & $\underline{\text { Trial type }}$ & Mean RT & $\underline{95 \% \mathrm{CI}}$ & $\underline{\underline{\mathbf{S E}}}$ \\
\hline Congruent & 486 & $472-499$ & 7 & Congruent & 488 & $574-502$ & 6 \\
\hline Neutral & 496 & $481-511$ & 7 & Neutral & 496 & $583-512$ & 7 \\
\hline Incongruent & 495 & $479-511$ & 8 & Incongruent & 500 & $484-516$ & 8 \\
\hline
\end{tabular}

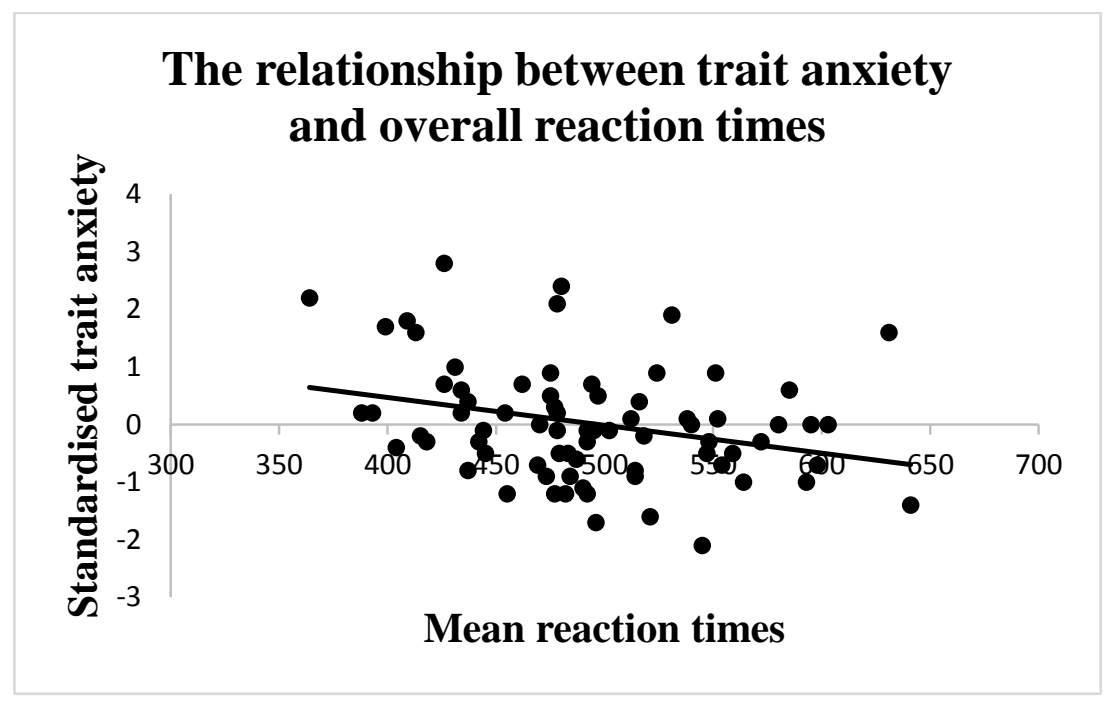

Figure 1: The negative correlation $(r=-0.3)$ between standardised trait anxiety and overall RTs. 
Table 2: Mean proportion correct (Prop/C), 95\% confidence intervals (95\%CI) and standard errors of the mean (SE) for each stimulus type.

\begin{tabular}{|c|c|c|c|c|c|c|c|}
\hline \multicolumn{4}{|c|}{ Happy target faces } & \multicolumn{4}{|c|}{ Fearful target faces } \\
\hline Trial type & Prop/C & $\underline{\underline{95 \%} \mathrm{CI}}$ & $\underline{\underline{\mathbf{S E}}}$ & Trial type & Prop/C & $\underline{\underline{95 \%} \mathrm{CI}}$ & $\underline{\underline{\mathbf{S E}}}$ \\
\hline Congruent & 0.97 & $0.97-0.98$ & 0.003 & Congruent & 0.97 & $0.96-0.97$ & 0.004 \\
\hline Neutral & 0.97 & $0.96-0.97$ & 0.004 & Neutral & 0.97 & $0.97-0.98$ & 0.004 \\
\hline Incongruent & 0.95 & $0.93-0.96$ & 0.007 & Incongruent & 0.94 & $0.92-0.95$ & 0.007 \\
\hline
\end{tabular}

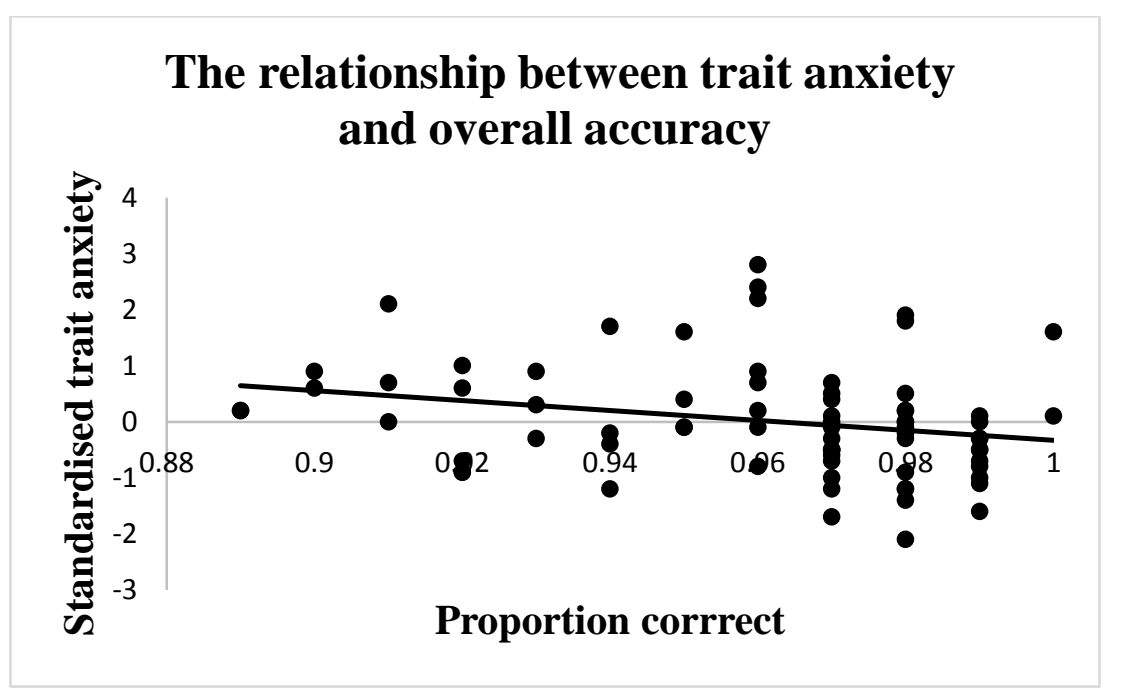

Figure 2: The negative correlation $(r=-0.25)$ between standardised trait anxiety and overall accuracy. 


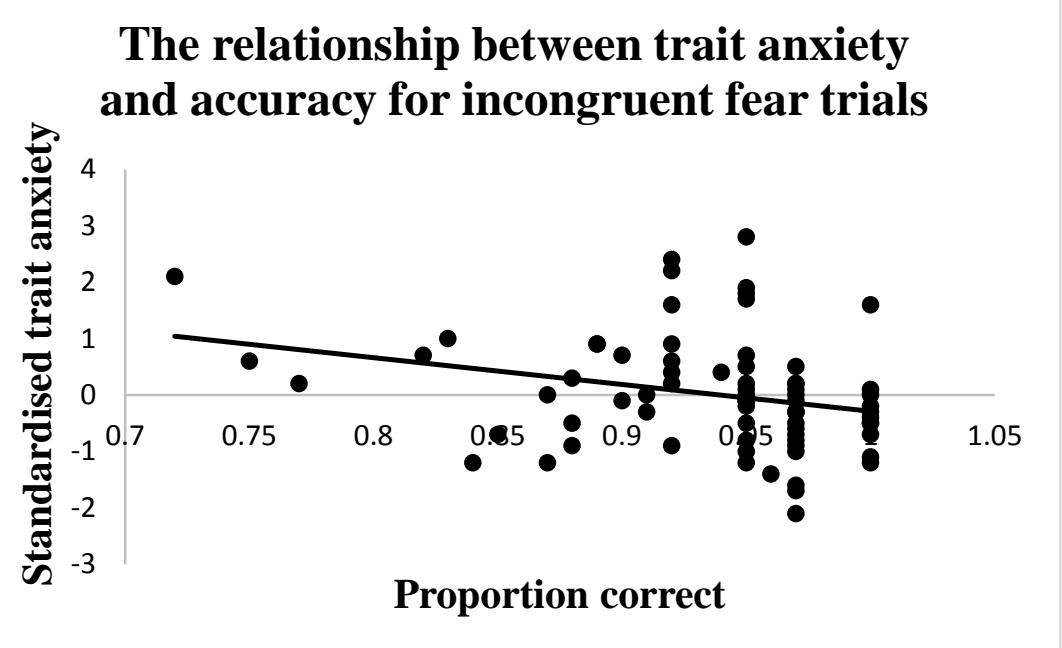

Figure 3: The negative correlation $(r=-0.28)$ between standardised trait anxiety and accuracy for incongruent fear trials. 